\title{
Study and Analysis of Photonic Crystal Fiber for Broadband Laser Source Design
}

\author{
Mohammed Faisal, SK. M. Jahangir Kabir and M. R. Karim
}

\begin{abstract}
Photonic Crystal Fiber (PCF) has been a topic of extensive research in the field of nonlinear fiber optics. It is due to its combination of linear and nonlinear properties which ultimately results in the phenomenon having various types of applications. One of the phenomena is supercontinuum generation that has been extensively studied numerically and experimentally over the last few decades as it has important applications in the field of medical imaging and sensing. In this paper, we numerically analysed and studied PCF of hexagonal core with various parameters and propose our novel 'Star in Hexagon' design as a good candidate for the broadband supercontinuum generation and hence a broadband laser source. We numerically pumped a long $10 \mathrm{~mm}$ PCF of this kind at $3.1 \mu \mathrm{m}$ wavelength with a pulse having a power of $4000 \mathrm{~W}$ and a temporal width of 50 fs. A broadband supercontinuum ranging from $1.7 \mu \mathrm{m}$ to $18 \mu \mathrm{m}$ was obtained. The generated spectrum is quite well in the mid-infrared region. Hence it can be utilised for Mid - Infrared (MIR) applications.
\end{abstract}

Index Terms-Hexagonal PCF, Novel PCF, Supercontinuum generation, MIR

\section{INTRODUCTION}

Photonic Crystal Fiber (PCF) has been an important research topic for the last few decades. It is due to some types distinguished properties that PCF possesses. There are two of guiding mechanism in PCF: indexguided microstructures and photonic band-gap fibers. In case of index-guided microstructure $\mathrm{PCF}$, it is found from previous research works that the geometrical design of the fiber governs various types of optical properties which include chromatic dispersion, confinement loss, effective area, and so on. Nonlinearity is one of the most important characteristic that PCFs showcase. This characteristic can be easily maneuvered with variations in the geometrical design and choice of material within the fiber. The nonlinear nature of PCFs results in the generation of Supercontinuum (SC) that has enormous applications such as optical coherence tomography, spectroscopy, frequency metrology, early cancer cell detection, hazardous material sensing, and so on.

Microstructured photonic crystal fiber is the type of PCF where air holes are structured longitudinally in a periodic manner down the fiber cladding. This type of PCF is also

Mohammed Faisal, Chittagong Independent University (CIU), Chattogram, Bangladesh (e-mail: mohammedfaisalciu@gmail.com).

SK. M. Jahangir Kabir, Chittagong Independent University (CIU), Chattogram, Bangladesh (e-mail: jahangirkabirmarinectg100@gmail.com).

M. R. Karim is the Dean, School of Science and Engineering, Chittagong Independent University (CIU), Chattogram, Bangladesh (e-mail: mrkarim@ciu.edu.bd). referred to as Holey PCF [1]. There is a greater index contrast in case of microstructured PCF than that of conventional fiber. A fundamental difference between index guided microstructure PCF and conventional fiber arises from the fact that the guided mode interacts with the cladding region and is dependent on wavelength [1]. This wavelength dependence results in a number of distinguished properties that can be optimized by manipulating the design of the microstructure PCF. A lot of research works have been reported earlier to accomplish improved designs by holding a tradeoff between different types of optical properties [2]. Effective refractive index is an important parameter in case of microstructured index guided PCF. This effective refractive index is usually retrieved in a complex form that varies with the wavelength of light in the PCF. The optical properties such as waveguide dispersion, confinement loss, and effective area also vary with the variation of the effective refractive index [2]. Previous research works testify that it is possible to optimize the optical properties through the variation in the air hole diameter and pitch of the designed PCF geometry [3]. Moreover, the effects of different types of air hole structure on the optical properties have been reported earlier [3-6]. Nonlinear optics is the analysis and the study of the phenomena that takes place when the optical properties of a material system vary nonlinearly with the presence of light. The discovery of the second-harmonic generation by Franken et al. (1961) followed by the demonstration of the first working laser by Maiman in 1960 paved the way to nonlinear optics. The word 'Nonlinear' is relevant in the sense that the optical properties of the material system varies nonlinearly with the strength of the optical field. The field of nonlinear fiber optics continued to flourish in the 1990s. PCF's have been introduced in the year of 1996 where it was shown that structural variations in these types of fibers affect the dispersive as well as nonlinear characteristics [7], [8], [9]. These nonlinear characteristics are very much dependent on the size of the core as well as the material of the PCF. Supercontinuum (SC) is a nonlinear effect that indicates the generation of extremely broad optical spectra which can be implemented and engineered through microstructured PCF fibers. This novel nonlinear property contributes to many sophisticated applications such as optical coherence tomography, high-resolution spectroscopy, food quality control, biomedical imaging, gas sensing, and so on. In case of microstructured photonic crystal fiber, the nonlinear 
characteristics can be varied and optimized through the variations of the microstructure design. Extensive research works have been performed to generate the broadband supercontinuum by designing various types of PCF with different types of materials. Sharma et al. [10] numerically presented a silica PCF of $15 \mathrm{~cm}$ with pump pulses of $50 \mathrm{fs}$. By applying $1 \mathrm{~kW}$ and $5 \mathrm{~kW}$ peak power they have achieved about $1200 \mathrm{~nm}$ and $1700 \mathrm{~nm}$ broadened supercontinuum. Begum F. et al. [11] proposed a highly nonlinear PCF with seven rings of air hole and silica material. In [11] they have presented supercontinuum spectrum from $960 \mathrm{~nm}$ to $1870 \mathrm{~nm}$. Research works have not been confined to only silica material. As nonlinearity also depends on the material that is incorporated in the PCF, extensive numerical and experimental studies have been performed by using different types of materials [12]. Karim et al. [13] numerically generated an ultraband supercontinuum in the mid-infrared region using dispersion engineered GeAsSe chalcogenide $(\mathrm{ChG})$ glass photonic crystal fiber. It has been presented in [13] that SC spectral broadening with flattened output can be obtained by variation in the pitch. By applying a peak power of $4 \mathrm{~kW}, 1.8 \mu \mathrm{m}$ to $14 \mu \mathrm{m}$ broadband supercontinuum has been generated [13]. Rahman et al. [14] numerically generated a $1.3 \mu \mathrm{m}$ to $11 \mu \mathrm{m}$ ultrabroadband midinfrared supercontinuum at a wavelength of $3.1 \mu \mathrm{m}$ with a peak power of $3 \mathrm{~kW}$. A $1 \mu \mathrm{m}$ to $10 \mu \mathrm{m}$ mid-infrared supercontinuum was numerically generated by using $100 \mathrm{~mm}$ long $\mathrm{Ge}_{11.5} \mathrm{As}_{24} \mathrm{Se}_{64.5} \mathrm{PCF}$ at a pump wavelength of $3.1 \mu \mathrm{m}$ with 85 fs pump pulses and peak power of $3 \mathrm{~kW} \mathrm{[15].} \mathrm{An}$ ultrabroadband mid-infrared supercontinuum of $1 \mu \mathrm{m}$ to $14 \mu \mathrm{m}$ has been reported with a $9 \mathrm{~mm}$ long $\mathrm{Ga}_{8} \mathrm{Sb}_{32} \mathrm{~S}_{60}$ chalcogenide PCF at $4.0 \mu \mathrm{m}$ pump wavelength with 90 fs laser pulse and peak power of $8.19 \mathrm{~kW}[16]$.

In this paper, three novel hexagonal core PCFs are presented which are numerically analyzed and compared with the conventional hexagonal core PCF. Proposed structure named as Star in Hexagon shows flat dispersion over a wide wavelength region. This PCF is further optimized to tailor dispersion and obtain zero dispersion wavelength close to the pump wavelength. The optimized PCF when numerically pumped at $3.1 \mu \mathrm{m}$ wavelength with a pulse having a power of $4000 \mathrm{~W}$ and a temporal width of 50 fs produces a broadband supercontinuum ranging from $1.7 \mu \mathrm{m}$ to $18 \mu \mathrm{m}$.

\section{THEORY OF NUMERICAL MODELLING}

The modal properties of the PCF are obtained by Finite Element Method (FEM). The wavelength dependent intrinsic refractive index of the chalcogenide material, $\mathrm{Ge}_{11.5} \mathrm{As}_{24} \mathrm{Se}_{64.5}$, is accounted by using the well-known Sellmeier Equation given as:

$$
n^{2}(\lambda)=1+\frac{5.78525 \lambda^{2}}{\lambda^{2}-0.28795^{2}}+\frac{0.39705 \lambda^{2}}{\lambda^{2}-30.39338^{2}}
$$

where $\lambda$ is the wavelength in micrometers.

The combined effect of material and waveguide dispersion is commonly called chromatic dispersion [17]. This is given by dispersion parameter $\mathrm{D}(\lambda)$ as:

$$
\mathrm{D}(\lambda)=-\frac{\lambda}{\mathrm{c}} \frac{\mathrm{d}^{2} \operatorname{Re}\left(\mathrm{n}_{\mathrm{eff}}\right)}{\mathrm{d} \lambda^{2}}
$$

where $c$ is the speed of light and $\mathrm{n}_{\mathrm{eff}}$ is the effective refractive index.

Confinement loss indicates light confinement ability of the optical fiber within its core region [18]. It is given as:

$$
L_{c}=8.686 \times \frac{2 \pi}{\lambda} \times \operatorname{Im}\left[\mathrm{n}_{\mathrm{eff}}\right]
$$

where $\operatorname{Im}\left[\mathrm{n}_{\text {eff }}\right]$ is the imaginary part of the effective refractive index.

Simulation of wave propagation in optical fiber requires the use of solving the Generalized Nonlinear Schrödinger Equation (GNLSE). This equation essentially accounts for the linear and nonlinear effects on wave propagation within a fiber $[19,20]$. It is given as:

$$
\begin{aligned}
\frac{\partial A}{\partial z}+\frac{\alpha}{2} A-\sum_{k \geq 2} \frac{i^{k+1}}{k !} \beta_{k} \frac{\partial^{k} A}{\partial T^{k}} \\
=i \gamma\left(1+i \tau_{\text {shock }} \frac{\partial}{\partial T}\right) \\
\quad \times\left(A(z, T) \int_{-\infty}^{\infty} R\left(T^{\prime}\right) \mid A(z, T\right. \\
\left.\left.-T^{\prime}\right)\left.\right|^{2} d T^{\prime}\right)
\end{aligned}
$$

where $A$ is the electrical field amplitude, $\alpha$ is the linear propagation loss, $\beta_{k}$ is the kth-order dispersion parameter associated with the Taylor series expansion of the propagation constant $\beta(\omega)$ about the central frequency $\omega_{0}, T=t-z / v_{g}$ is the retarded time frame moving with the group velocity $v_{g}=$ $1 / \beta_{1}\left(\omega_{0}\right)$ at $\omega_{0}$ and $\gamma$ is the nonlinear coefficient. The left-hand side of GNLSE models linear propagation effects, while the right-hand side models nonlinear effects on pulse propagation. $\alpha$ is taken as $0.5 \mathrm{~dB} / \mathrm{cm}$ as reported [21-23]. The material response function $R(t)$ is given as:

$$
\begin{gathered}
R(t)=\left(1-f_{r}\right) \delta(t)+f_{r} h_{r}(t) \\
h_{r}(t)=\frac{\tau_{1}^{2}+\tau_{2}^{2}}{\tau_{1}} \exp \left(-\frac{t}{\tau_{2}}\right) \sin \left(\frac{t}{\tau_{1}}\right)
\end{gathered}
$$

where $f_{R}=0.031, \tau_{1}=15.5$ fs and $\tau_{2}=230.5$ fs for $\mathrm{Ge}_{11.5} \mathrm{As}_{24} \mathrm{Se}_{64.5}[13,24]$.

For the study of supercontinuum generation, we numerically simulate the GNLSE provided in Eq. (4). $A(z, T)$ represents the complex envelope of the propagating field in a frame of reference traveling at the group velocity of the input pulse. We consider an input pulse having a central angular frequency $\omega_{0}$ and hyperbolic-secant shape [25]:

$$
\begin{gathered}
A(0, T)=\sqrt{P_{0}} \operatorname{sech}\left(\frac{T}{T_{0}}\right) \\
T=t-\frac{z}{v_{g}\left(\omega_{0}\right)}
\end{gathered}
$$


where $T_{0}$ is the input pulse duration, $P_{0}$ is the input pulse peak power and $v_{g}$ is the group velocity at frequency $\omega_{0}$.

Equation (4) can be written as:

$$
\frac{\partial A(z, T)}{\partial z}=(\widehat{D}+\widehat{N}) A(z, T)
$$

where $\widehat{D}$ represents the linear term and $\widehat{N}$ represents the nonlinear term.

Numerical solutions of Equation (4) are obtained by using the Split-Step Fourier method (SSFM) through which it is possible to solve the linear part of the equation completely in the frequency domain while the nonlinear part is solved partially in the time domain and partially in the frequency domain [26]. SSFM provides the linear part and the nonlinear part in the Ordinary Differential Equation (ODE) form where they are solved using fourth-order Runge-Kutta integration method [27, 28], obtaining $A(z+d z, T)$.

In our simulations we use $2^{13}$ points to discretize a temporal window which is much larger than the input pulse duration $T_{0}$. The other key parameter that has to be set is the fiber discretization step $\Delta z$ which can be characterized by $z / n$ where $n$ is a number signifying the discretization step. Presence of higher-order dispersion terms tends to produce numerical instabilities [29] that appear in the spectrum as pronounced peaks at fixed frequencies. By appropriately reducing $\Delta z$ it is possible to shift the instability peaks outside the spectral window, so avoiding unwanted interferences. We set $n$ to be about 200, which was consistent with results obtained.

\section{DESIGN PRINCIPLE}

Initially the conventional hexagonal PCF is designed. This design is shown in Fig. 1. The core is hexagonal with a defect in the center which allows light propagation. The cladding is taken hexagonal with uniform air holes. This structure is analyzed with $\mathrm{Ge}_{11.5} \mathrm{As}_{24} \mathrm{Se}_{64.5}$ as the material.

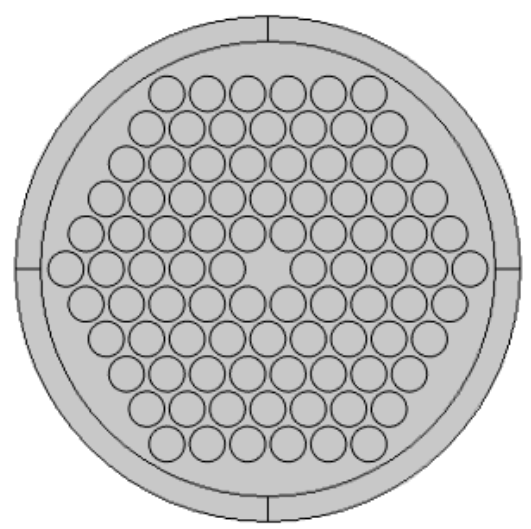

Fig. 1: A schematic cross section of the hexagonal PCF [13].

We proposed three novel designs. The schematic diagram of the structures are depicted in Fig. 2.

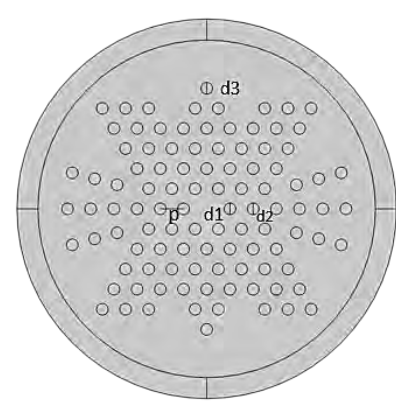

(a)

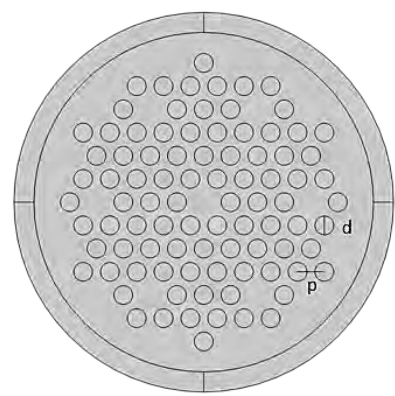

(b)

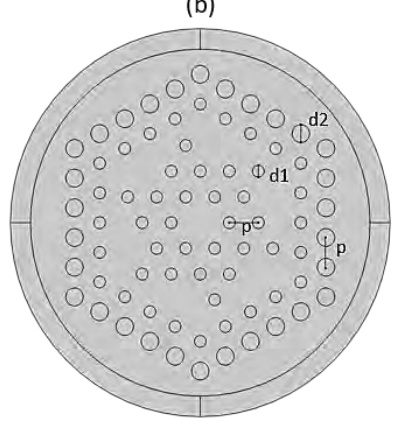

(c)

Fig. 2: Schematic cross section of the novel PCF (a) Beetles (b) Metatron (c) Star in Hexagon.

All the designs have a hexagonal core. The difference lies in the cladding of the structure. Fig. 2(a) shows the 'Beetles' design. The cladding of this design resembles a 'Beetles' structure and hence the name has been given. The first inner layer has an air hole diameter represented as $\mathrm{d} 1$. The three feather like branches protruding from the structure has an air hole diameter represented as $\mathrm{d} 3$. The rest of the structure has a uniform air hole diameter represented as $\mathrm{d} 2$. The pitch is constant throughout the structure which is denoted as p. Fig. 2(b) shows the 'Metatron' design. The core is hexagonal as usual. The term 'Metatron' has been derived from a jewish scripture as the structure has a close similarity. The core section is replicated around the structure. There are five replica of the core section all around the structure. The air hole diameter is denoted as $d$ whereas pitch is represented as p. Fig. 2(c) shows the third proposed structure. The proposed structure has similarity in the first two inner rings with the conventional hexagonal PCF. Modification is prominent after the two rings. Six single air holes connect the inner structure with the outermost two layers of hexagonal structure. This leaves vacant spaces after the second inner layer. The inner structure somewhat looks like a star and hence the structure is named as 'Star in Hexagon.' Air hole grating is applied to the proposed 
structure. The air hole diameter of the outermost ring, d2, and the air hole diameter of the rest of the structure, $\mathrm{d} 1$, is grated.

The geometrical parameters are varied for desired dispersion profile for SC generation. This is compared with five ring conventional hexagonal structure. The PCFs are designed in the COMSOL Multiphysics 5.5 software which uses Finite Element Method (FEM) to conduct analysis [30-32].

\section{RESULT}

Initially the proposed structures are set to geometrical parameters as reported from the conventional five layer hexagonal structure in [13]. This approach is adopted to aid an ease in comparative analysis between the conventional and the proposed structures. Table I shows the geometrical parameters of the novel designs.

TABLE I

GEOMETRICAL PARAMETERS OF THE NOVEL DESIGNS

\begin{tabular}{ll}
\hline \hline \multicolumn{1}{c}{ Design } & \multicolumn{1}{c}{ Geometrical Parameters } \\
\hline Beetles & $\Lambda=2.2 \mu \mathrm{m}, \mathrm{d} 1=\mathrm{d} 2=\mathrm{d} 3=1.1 \mu \mathrm{m}$ \\
Metatron & $\mathrm{d}=1.2 \mu \mathrm{m}, \Lambda=2.4 \mu \mathrm{m}$ \\
Star in Hexagon & $\mathrm{d} 1=1.2 \mu \mathrm{m}, \mathrm{d} 2=1.44 \mu \mathrm{m}, \Lambda=2.4 \mu \mathrm{m}$ \\
\hline \hline
\end{tabular}

We have analyzed the dispersion of the three new designs presented with the geometrical parameters specified in Table 1 . Fig. 3 shows the dispersion of the proposed structures over a wide wavelength range. To get a broadband SC it is necessary to get a flat nearly zero dispersion curve in the anomalous dispersion region. It is also necessary to have a very low dispersion in the vicinity of the pump wavelength.

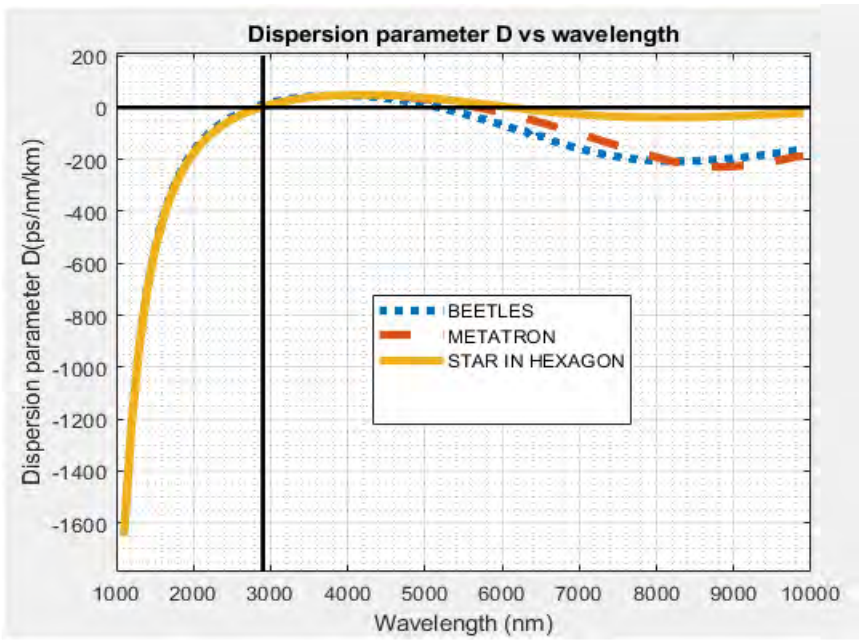

Fig. 3: Combined dispersion curves of the 'Beetles', 'Metatron' and 'Star in Hexagon' design.

Fig. 3 shows that the dispersion curve of the proposed designs where the 'Star in Hexagon' design gives better flat dispersion curve than the other two designs. To get a broadband $\mathrm{SC}$ it is necessary to get a flat nearly zero dispersion curve in the anomalous dispersion region. It is also necessary to have a very low dispersion in the vicinity of the pump wavelength. Hence the Star in Hexagon can be optimized to get a long and flat nearly zero dispersion in the anomalous region. The comparison between the anomalous dispersion regimes of proposed structures are presented in Table II.

TABLE II

COMPARISON OF THE ANOMALOUS DISPERSION REGIMES OF THE NOVEL DESIGNS

\begin{tabular}{lcc}
\hline \hline \multicolumn{1}{c}{ Design } & Anomalous & Bandwidth of Anomalous \\
& Dispersion Regime & \begin{tabular}{c} 
Dispersion \\
\hline Beetles
\end{tabular} \\
Metatron & $2800 \mathrm{~nm}-5100 \mathrm{~nm}$ & $2300 \mathrm{~nm}$ \\
Star in Hexagon & $2900 \mathrm{~nm}-5600 \mathrm{~nm}$ & $2700 \mathrm{~nm}$ \\
\hline \hline
\end{tabular}

Having superior characteristics over the other designs, Star in Hexagon is compared with the conventional hexagonal structure. Fig. 4 shows the mode profile of both the 'Traditional Hexagonal' and the 'Star in Hexagon' design. Although the core is identical, the outer layers are different. There is graded air hole diameter employment in the Star in Hexagon design. Also the proposed design has a fewer number of air holes in comparison. This is favorable for fabrication. The mode profile seems to be identical, but the superior properties of the proposed design are well revealed in the dispersion analysis. Fig. 5 shows the dispersion curves of the 'Traditional Hexagonal' design and 'Star in Hexagon' design.

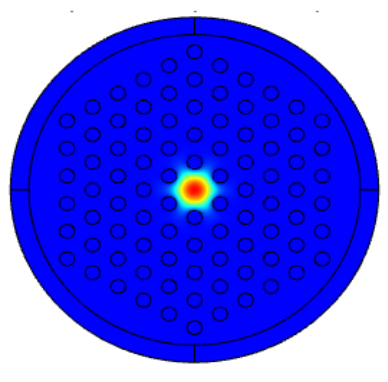

(a)

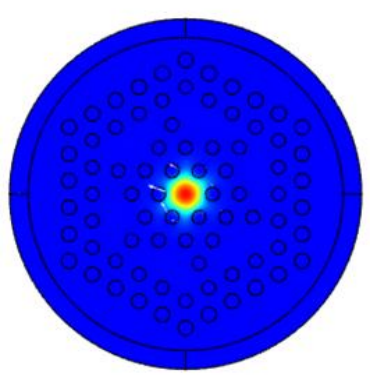

(b)
Figure 4: Mode profile - (a) Traditional Hexagonal [13] (b) Star in Hexagon design.

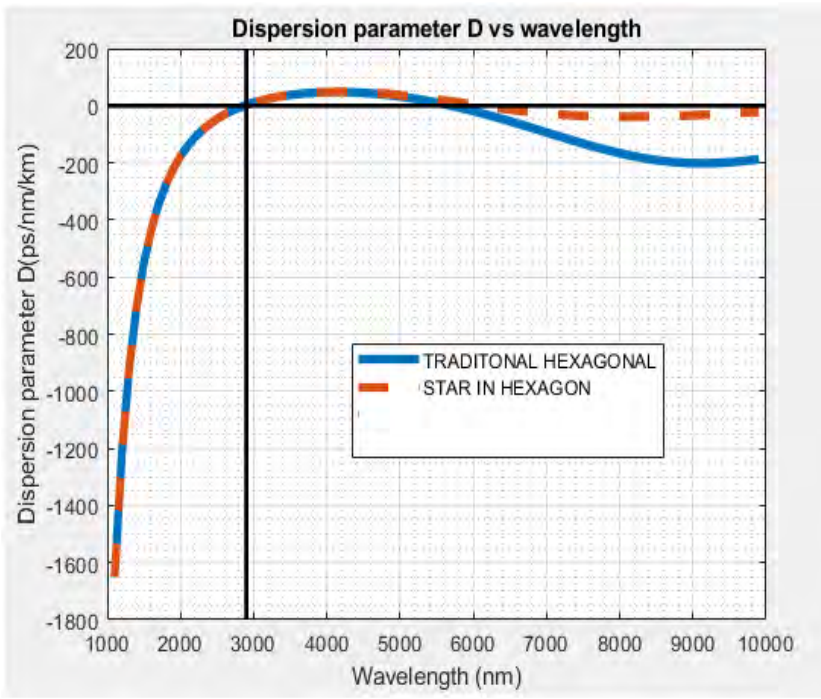

Fig. 5: Dispersion curve of the 'Traditional Hexagonal' design and 'Star in Hexagon’ design. 
We observe from the Fig. 5 that the anomalous dispersion regime of the proposed structure extends from $2900 \mathrm{~nm}$ to 6000 $\mathrm{nm}$ with a bandwidth of $3100 \mathrm{~nm}$ whereas the traditional design has an anomalous dispersion regime extending from $2900 \mathrm{~nm}$ to $5600 \mathrm{~nm}$ with a bandwidth of $2700 \mathrm{~nm}$. It is evident that the 'Star in Hexagon' has a greater anomalous dispersion which is important criteria for broadband SC generation. Hence, there is an opportunity of having a long anomalous dispersion region in 'Star in Hexagon' design as the dispersion curve is less convex than the dispersion curve of the 'Traditional Hexagonal' design. The anomalous dispersion regimes of all the analyzed structures has been summarized in Table III.

TABLE III

COMPARISON OF THE ANOMALOUS DISPERSION REGIMES OF THE NOVEL AND TRADITIONAL DESIGNS

\begin{tabular}{lcc}
\hline \hline \multicolumn{1}{c}{ Design } & $\begin{array}{c}\text { Anomalous } \\
\text { Dispersion Regime }\end{array}$ & $\begin{array}{c}\text { Bandwidth of Anomalous } \\
\text { Dispersion }\end{array}$ \\
\hline Traditional & $2900 \mathrm{~nm}-5600 \mathrm{~nm}$ & $2700 \mathrm{~nm}$ \\
Hexagonal [13] & & \\
Beetles & $2800 \mathrm{~nm}-5100 \mathrm{~nm}$ & $2300 \mathrm{~nm}$ \\
Metatron & $2900 \mathrm{~nm}-5600 \mathrm{~nm}$ & $2700 \mathrm{~nm}$ \\
Star in Hexagon & $2900 \mathrm{~nm}-6000 \mathrm{~nm}$ & $3100 \mathrm{~nm}$ \\
\hline \hline
\end{tabular}

Next we have a look at the confinement loss of the two structures. Fig. 6 shows the confinement loss of both the 'Traditional Hexagonal' and 'Star in Hexagon' design. It is evident that the confinement loss is quite the same for both the designs.

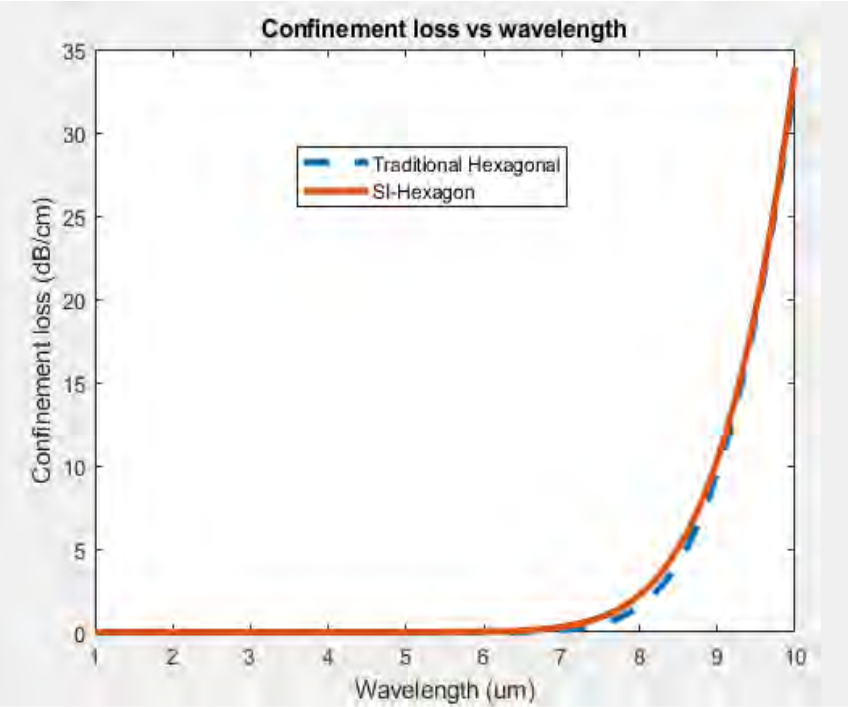

Fig. 6: Confinement loss of 'Traditional Hexagonal' and 'Star in Hexagon' design.

From the above analysis we can elect 'Star in Hexagon' as a competitor to the conventional hexagonal structure with the proposed structure having superior characteristics.

Now we further optimize the 'Star in Hexagon' design in order to prepare it for broadband SC generation. Fig. 7 shows the geometrical variations of the proposed structure.

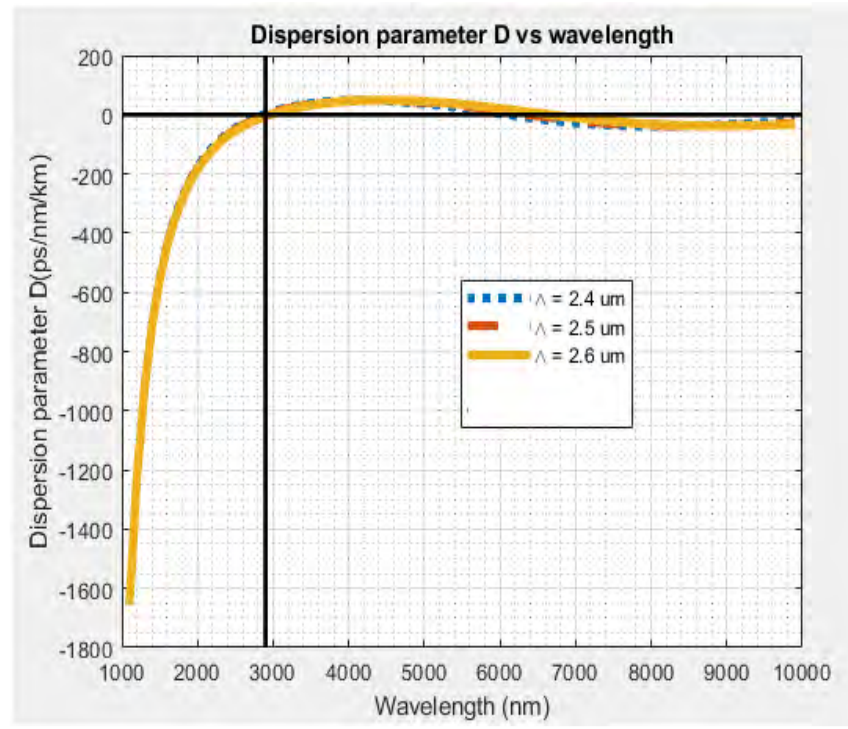

Fig. 7: Optimization of the dispersion curve through the pitch variation $\Lambda=$ $2.4 \mu \mathrm{m}, 2.5 \mu \mathrm{m}$, and $2.6 \mu \mathrm{m}$ keeping the inner ration $\mathrm{d} 1 / \Lambda=0.5$ constant and varying the outermost layer ratio $\mathrm{d} 2 / \Lambda$.

We have varied the pitch of the inner star structure as $2.4 \mu \mathrm{m}$, $2.5 \mu \mathrm{m}$ and $2.6 \mu \mathrm{m}$. In order to keep the inner ratio $\mathrm{d} 1 / \Lambda$ constant at 0.5 , we have varied the air hole diameter as $1.2 \mu \mathrm{m}$, $1.25 \mu \mathrm{m}$ and $1.3 \mu \mathrm{m}$. But we did not change the air hole diameter of the outermost hexagon layer. The diameter of the outermost layer is kept constant at $1.44 \mu \mathrm{m}$. As a result we get a varying $\mathrm{d} 2 / \Lambda$ ratio in relation to the varying pitch as $0.6,0.576$ and 0.553 . We observe that in case of the pitch $\Lambda=2.6 \mu \mathrm{m}$ we get the anomalous dispersion from $2900 \mathrm{~nm}$ to $6600 \mathrm{~nm}$. We get a bandwidth of $3700 \mathrm{~nm}$ which was $3100 \mathrm{~nm}$ before optimization. The dispersion in the anomalous regime is below $50 \mathrm{ps} / \mathrm{nm} / \mathrm{km}$. As a result we get a dispersion curve at the geometrical parameters of $\mathrm{d} 1=1.3 \mu \mathrm{m}, \mathrm{d} 2=1.44 \mu \mathrm{m}$ and pitch $\Lambda=2.6 \mu \mathrm{m}$ which has a wider anomalous dispersion in respect to other two curves.

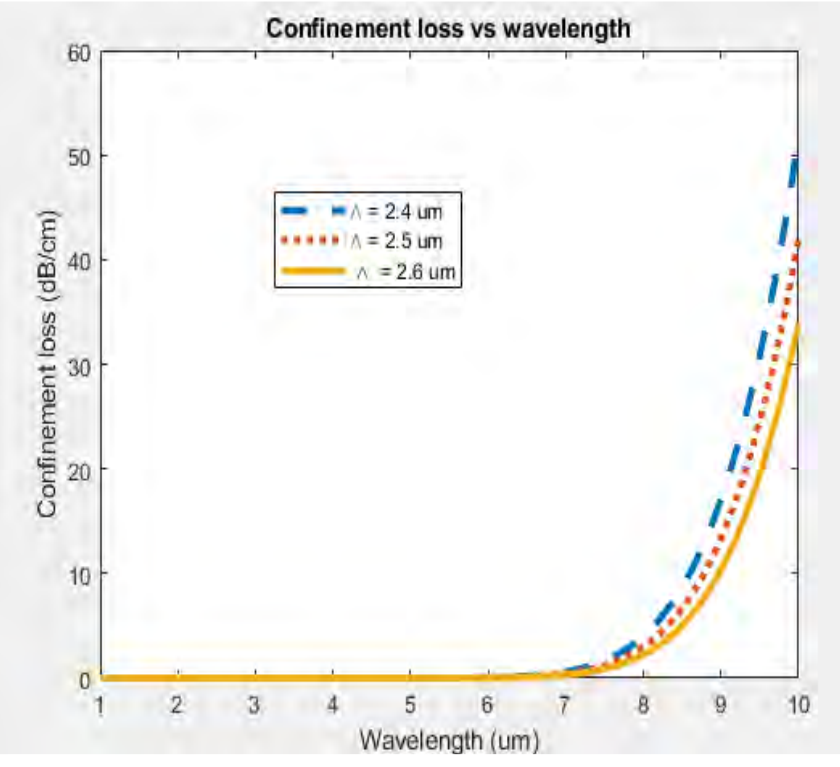

Fig. 8: Confinement loss of the 'Star in Hexagon' design for a variation in pitch. 


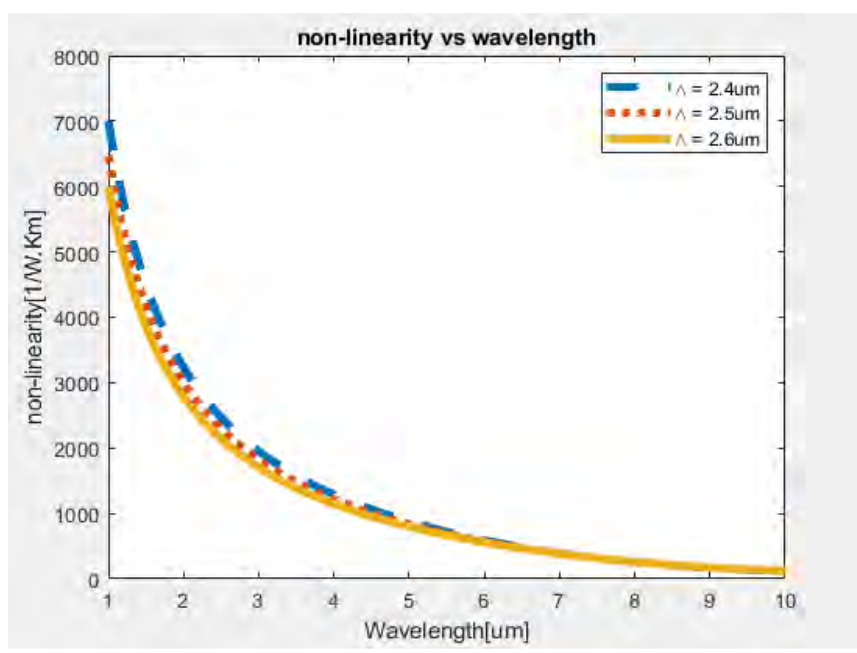

Fig. 9: Nonlinear curve of the three 'Star in Hexagon' for a variation in pitch.

The combined confinement loss is observed in Fig. 8 where the loss tends to decrease as we increase the pitch towards 2.6 $\mu \mathrm{m}$. From Fig. 9 we observe the nonlinearity as pitch is increased. There is no big change in the nonlinearity with the variations.

Supercontinuum (SC) has become one of the most significant topics in the field of nonlinear fiber optics. In optics, a SC is formed when a collection of nonlinear processes act together upon a pump beam in order to cause severe spectral broadening of the original pump beam. This SC has enormous applications in the field of medical imaging and different types of sensing applications.

We select the 'Star in Hexagon' with a pitch of $2.6 \mu \mathrm{m}$, air hole diameter $\mathrm{d} 1=1.3 \mu \mathrm{m}$ and outermost layer diameter $\mathrm{d} 2=$ $1.44 \mu \mathrm{m}$ for SC generation. We have performed simulations with the 'The Star in Hexagon' design at the $3.1 \mu \mathrm{m}$ wavelength, located in the mid-infrared region. Generated SC is analysed by launching pulses at different power levels and pulse durations. We first analyze the SC with power variations keeping the pulse width constant. Later on, we analyze the SC with pulse width variations keeping the power constant. In all our analysis the fiber length is kept constant to a value of 10 $\mathrm{mm}$. We know that low anomalous dispersion at pump wavelength is necessary in order to get a broadband SC [30].So we have not analyzed further with the longer pump wavelength.

SC generation is accomplished by solving the GNLSE with slowly varying envelope approximation. We numerically pumped the 'Star in Hexagon' of $10 \mathrm{~mm}$ length at $3.1 \mu \mathrm{m}$ wavelength by varying pulse power from $2000 \mathrm{~W}$ to $5000 \mathrm{~W}$ with a constant temporal width of 50 fs. Fig. 10 shows SC spectral evolution and Fig. 11 shows the corresponding spectral density evolution plot.

We then numerically pumped the 'Star in Hexagon' of 10 $\mathrm{mm}$ length at $3.1 \mu \mathrm{m}$ wavelength by varying the temporal width of the pulse from 30 fs to $200 \mathrm{fs}$ with a constant pulse power of 4000 W. Fig. 12 shows SC spectral evolution and Fig. 13 shows the corresponding spectral density evolution plot.
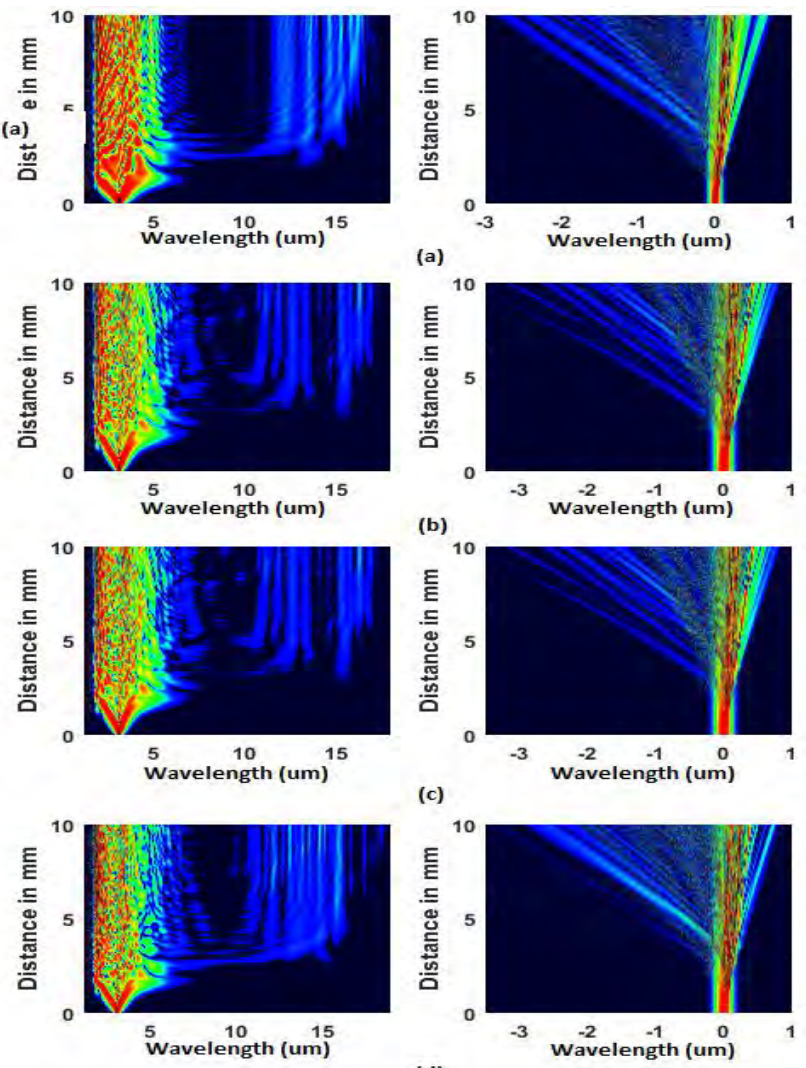

(d)

Fig. 10: Spectral density evolution plots with power variation (a) $2000 \mathrm{~W}$ (b) $3000 \mathrm{~W}$ (c) $4000 \mathrm{~W}$ (d) $5000 \mathrm{~W}$.

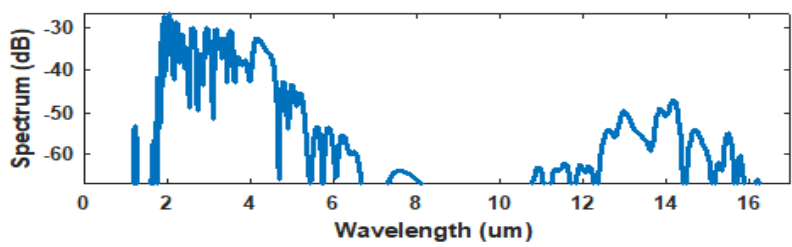

(a)

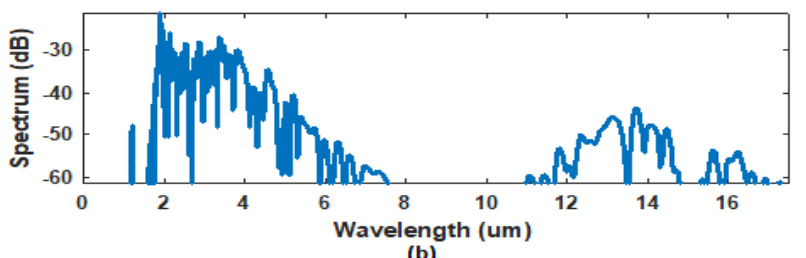

(b)

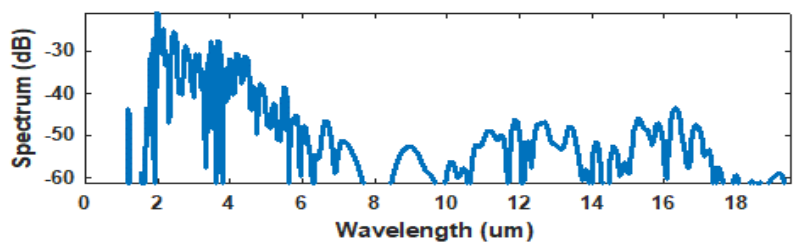

(c)

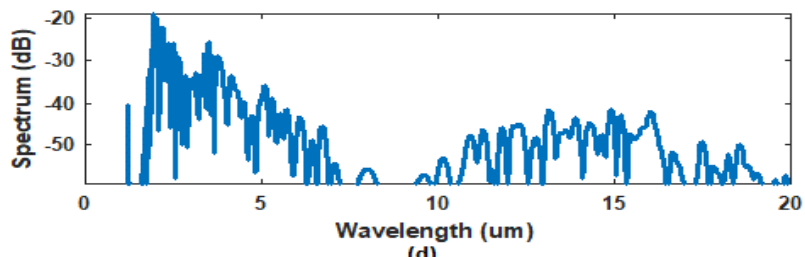

(d)

Fig. 11: SC spectral evolution plots with power variation (a) $2000 \mathrm{~W}$ (b) 3000 $\mathrm{W}$ (c) $4000 \mathrm{~W}$ (d) $5000 \mathrm{~W}$ 

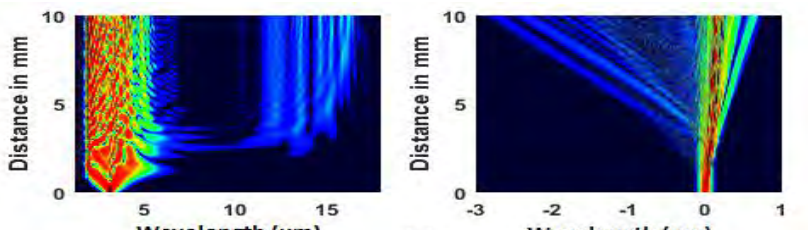
Wavelength (um)
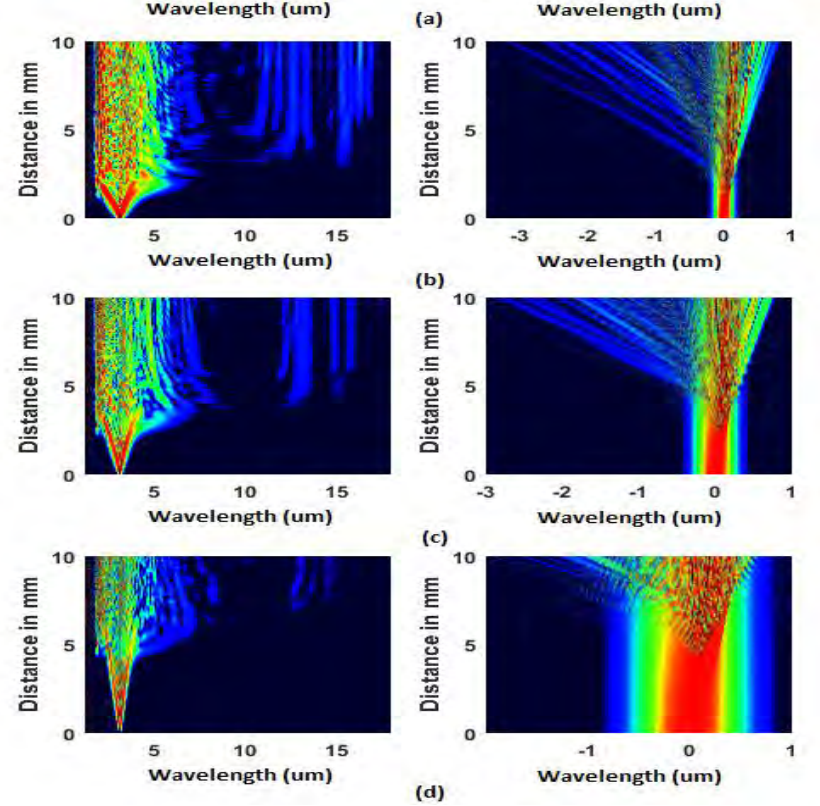

Fig. 12: Spectral density evolution plots with pulse width variation (a) $30 \mathrm{fs}$ (b) $50 \mathrm{fs}$ (c) $100 \mathrm{fs}$ (d) $200 \mathrm{fs}$.

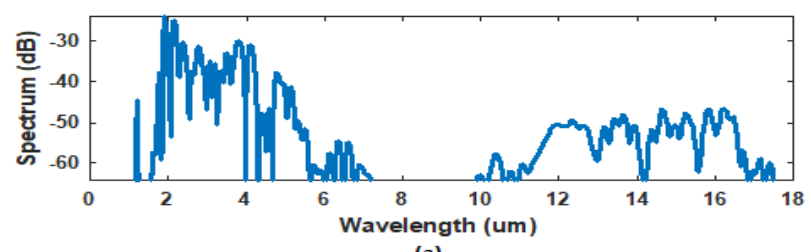

(a)

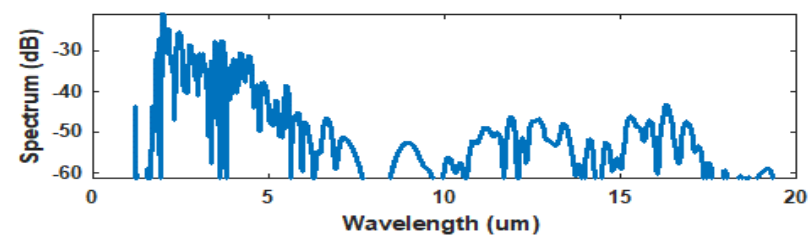

(b)

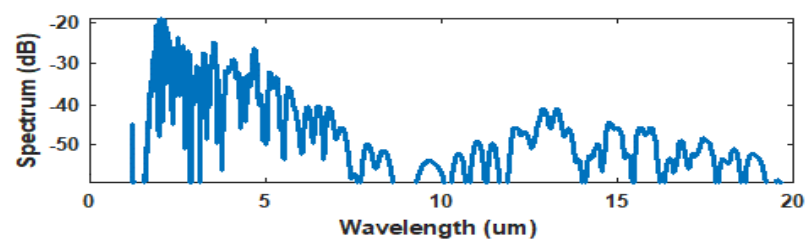

(c)

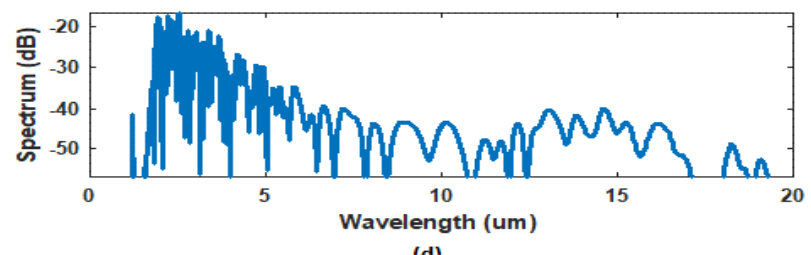

(d)

Fig. 13: SC spectral evolution plots with pulse width variation (a) $30 \mathrm{fs}$ (b) 50 fs (c) $100 \mathrm{fs}(\mathrm{d}) 200 \mathrm{fs}$.
Initially Self - Phase Midulation (SPM) and anomalous dispersion interacts to produce a self-reinforcing solitary wave known as soliton which remains unchanged after propafationg a long distance [19-20]. This is observed in Fig. 10 as the pulse propagates in the first $2.5 \mathrm{~mm}$ length. Perbutations which include the higher order dispersion and raman scattering then breaks up the solitons into a series of sub pulses. These sub pulses acts as pump in the raman gain region and are red shifted. This is observed in Fig. 10 as the increase in the higher wavelength spectral components with pulse propagation. This red shift also increases as the pump power is increased from $2000 \mathrm{~W}$ to $5000 \mathrm{~W}$. The red shift also causes a shedding of energy in opposite side resulting in blue shift [19-20]. This is well observed in the temporal evolution plot in Fig. 10 after the $2.5 \mathrm{~mm}$ length pulse evolution. Fig. 11 depicts that when we apply $2000 \mathrm{~W}$ and $3000 \mathrm{~W}$ peak power pulse, we get a spectrum that extends up to $16 \mu \mathrm{m}$ with a dip between $8.0 \mu \mathrm{m}$ and $12 \mu \mathrm{m}$. But in case of $4000 \mathrm{~W}$ and $5000 \mathrm{~W}$ the dip is improved and the spectrum extends beyond the $16 \mu \mathrm{m}$. Hence increase in pump power leads to broadband SC generation.

For the variation in the temporal width of pump pulse, a drastic change has been observed in Fig. 12 and 13. In case of $30 \mathrm{fs}$ pulse, the spectrum extends up to $16 \mu \mathrm{m}$ with a dip from $6.5 \mu \mathrm{m}$ up to $10.5 \mu \mathrm{m}$ and a characteristics soliton fission length [19-20] to about $0.5 \mathrm{~mm}$. In case of $50 \mathrm{fs}$ pump pulse, the spectrum is broadened extending up to $18 \mu \mathrm{m}$ with an improvement in the dip encountered previously. However it is notable that although the dip has improved, the long wavelength components have low power. The improvement in the dip of the spectrum is also observed for $100 \mathrm{fs}$ and $200 \mathrm{fs}$ pulse but with a loss of power in long wavelength components. The reason behind this is pretty clear from Fig. 12. We know that as we approach the picosecond pump pulses, soliton dynamics tend to cease. This is evident in the spectrum where we can see that soliton fission length has increased noticeably.

Finally, from all the analysis it is evident that we obtain a substantial supercontinuum ranging from $1.7 \mu \mathrm{m}$ to $18 \mu \mathrm{m}$ when the designed PCF 'The Star in Hexagon' is pumped at 3.1 $\mu \mathrm{m}$ with a $4000 \mathrm{~W} 50 \mathrm{fs}$ pulse. The generated spectrum is quite well in the mid-infrared region. Hence it can be utilised for MIR applications. However, one has also look whether the pump sources are available at the specified parameters. It has been found that pump sources at these configurations are not quiet currently available in the market. But, these pump sources are experimentally achievable. Lasers employing Optical Parametric Oscillator (OPO), Optical Parametric Amplifier (OPA), parallel amplifier channel and mode locking techniques had been reported to produce pump sources about $3 \mu \mathrm{m}$ with optimal power level for SC generation [33-38]. Synchronously pumped OPOs and Optical Parametric Chirped-Pulse Amplifiers (OPCPA) have produced good results with wavelength tuning capability. They are capable to produce $\mathrm{kW}$ range femtosecond output about $3 \mu \mathrm{m}$ for a high power input source at about $1.55 \mu \mathrm{m}$ wavelength [39-40]. Hence the numerically generated supercontinuum with 'The Star in Hexagon' design can be practically implemented. 


\section{CONCLUSION}

In our work we have numerically simulated our three novel hexagonal core PCFs. These proposed structures were compared with the conventional hexagonal core PCF. Geometrical parameter variation on dispersion was observed. Hence we proposed a hexagonal PCF named as 'Star in Hexagon' PCF which had some amazing characteristics with regard to the conventional structure.

Our proposed 'Star in Hexagon' PCF showed a low dispersion compared to the conventional hexagonal core PCF. We optimized the structure to increase the anomalous dispersion regime and hence flatten the curve. It showed a flat dispersion ranging from $2900 \mathrm{~nm}$ to $6600 \mathrm{~nm}$. But in case of the conventional structure it only spanned from $2900 \mathrm{~nm}$ to 5600 $\mathrm{nm}$. Hence we proposed the novel 'Star in Hexagon' PCF as a good candidate for the broadband laser design. A broadband SC ranging from $1.7 \mu \mathrm{m}$ to $18 \mu \mathrm{m}$ was obtained when the designed PCF 'The Star in Hexagon' was numerically pumped at $3.1 \mu \mathrm{m}$ with a pulse having a power of $4000 \mathrm{~W}$ and a temporal width of $50 \mathrm{fs}$. The generated spectrum is quite well in the mid-infrared region. Hence it can be utilised for MIR applications.

The proposed structure may find a good application in chromatic dispersion control or dispersion shifted fiber. More extensive dispersion tailoring can give more amazing results with a good possibility of broadband laser source outcome. One can also include the wavelength dependent nonlinearity and loss due to attenuation. This will improve the reliability of the study. We will further continue to work on the designs proposed to nurture it for various applications.

\section{REFERENCES}

[1] John M. Dudley, Goëry Genty, and Stéphane coen, "Supercontinuum generation in photonic crystal fiber," Reviews of Modern Physics, vol. 78, December, 2006.

[2] Md. Bellal Hossain, Abdullah Al-Mamun Bulbul, Md. Abdul Mukit, and Etu Podder, "Analysis of Optical Properties for Square, Circular and Hexagonal Photonic Crystal Fiber," in Optics and Photonics Journal, vol. 7, no. 11, 2017.

[3] Sagor Biswas, Ragib Shakil Rafi, Md. Abdullah Al-Amin, and Sabbir Alam, "Analysis of the Effect of Air Hole Diameter and Lattice Pitch in Optical Properties for Hexagonal Photonic Crystal Fiber, " in Optics and Photonics Journal, vol. 5, no. 7, 2015.

[4] S. M. A. Razzak, Yoshinori Namihira, Feroza Begum, Shubi Kaijage, Tatsuya Kinjo, Jitsuryo Nakahodo, Kazuya Miyagi, and Nianyu Zou, "Decagonal Photonic Crystal Fibers with Ultra-Flattened Chromatic Dispersion and Low Confinement Loss," OFC/NFOEC 2007 Conference on Optical Fiber Communication and the National Fiber Optic Engineers Conference, Anaheim, CA, 2007, pp. 1-6.

[5] S. M. A. Razzak and Yoshinori Namihira, "Tailoring Dispersion and Confinement Losses of Photonic Crystal Fibers Using Hybrid Cladding," in Journal of Lightwave Technology, vol. 26, no. 13, pp. 1909-1914, July $1,2008$.

[6] Pooja Agarwal, "Modeling of Elliptical Air Hole PCF for Lower Dispersion," Advances in Electronic and Electric Engineering, vol. 3, no. 4, pp. 439-446, 2013.

[7] R. Buczynski, "Photonic Crystal Fibers," in Acta Physica Polonica A, vol. 106, no. 2, 2004.

[8] Arpit Singh Yadav, Aparna Singh, Vijay Shanker Chaudhary, and Dharmendra Kumar, "Ultra-Flat Dispersion with High Nonlinearity Hexagonal Photonic Crystal Fiber," 2018 5th IEEE Uttar Pradesh Section International Conference on Electrical, Electronics and Computer Engineering (UPCON), Gorakhpur, 2018, pp 1-3.
[9] K. Saitoh, M. Koshiba, T. Hasegawa, and E. Sasaoka, "Chromatic dispersion control in photonic crystal fibers: application to ultra-flattened dispersion," Optics Express, vol. 11, issue 8, pp. 843-852, 2003.

[10] Mohit Sharma, Swapan Konar, and Kaisar R. Khan, "Supercontinuum generation in Highly nonlinear hexagonal photonic crystal fiber at very low power," Journal of Nanophotonics, vol. 9, no. 1, April, 2015.

[11] Feroza Begum, Hazwani Suhaimi, Norazanita Shamsuddin, Martin Geoffrey Blundell, and Yoshinori Namihira, "Supercontinuum generated high power highly Nonlinear photonic crystal fiber for medical and optical communications applications," Modern Electronic Materials, vol.4, no. 2, pp. 53-58, June, 2018.

[12] Shixun Dai, yingying Wang, xuefeng Peng, Peiqing Zhang, Xunsi Wang, and Yinsheng Xu, "A review of Mid-infrared Supercontinuum Generation in Chalcogenide Glass Fibers," Applied Sciences, vol. 8, no. 5, pp 707, May 2018.

[13] H. Ahmad, M. R. Karim, S. Ghosh, and B. M. A. Rahman, "Generation of an ultrabroadband supercontinuum in the mid-infrared region using dispersion-engineered GeAsSe photonic crystal fiber," Optical and Quantum Electronics, vol. 50, no. 405, 2018.

[14] M. R. Karim, B. M. A. Rahman, Y. O. Azabi, A. Agrawal, and Govind P. Agrawal, "Ultrabroadband mid-infrared supercontinuum generation through dispersion engineering of chalcogenide microstructured fibers", Journal of the Optical Society of America B, vol. 32, no. 11, 2015.

[15] Sandeep Vyas, Takasumi Tanabe, Manish Tiwari, and Ghanshyam Singh, "Ultraflat broadband supercontinuum in highly nonlinear Ge11.5As24Se64.5 photonic crystal fibers," Ukranian Journal of Physical Optics, vol. 17, no. 3, pp 132-139, August, 2016.

[16] Pooja Chauhan, Ajeet Kumar, and Yogita Kalra, "Mid-infrared broadband supercontinuum generation in a highly nonlinear rectangular core chalcogenide photonic crystal fiber," Optical Fiber Technology, vol. 46, pp. 174-178, December, 2018.

[17] Lingxiao Fan, Lijun Xu, Hongxing Cai , Xiuping Sun, Fangzhou Zhao, Zhenjiang Li, and Zhenshan Qi, "Study on dispersion of photonic crystal fiber," in 2015 International Conference on Manipulation, Manufacturing and Measurement on the Nanoscale (3M-NANO), pp. 210-213, October, 2015.

[18] Md. Mahbub Hossain and Md. Maniruzzaman, "Study of Confinement Loss in Photonic Crystal Fiber," in International Conference on Materials, Electronics \& Information Engineering, June, 2015.

[19] Govind P. Agrawal, Nonlinear Fiber Optics, Fifth Edition, Oxford, UK: Academic Press, 2013.

[20] J. M. Dudley and J. R. Taylor, Supercontinuum Generation in Optical Fibers, Cambridge, United Kingdom: Cambridge University Press, 2010.

[21] M. R. Karim, B. M. A. Rahman, and Govind P. Agrawal, "Mid-infrared supercontinuum generation using dispersion-engineered $\mathrm{Ge}_{11.5} \mathrm{As}_{24} \mathrm{Se}_{64.5}$ chalcogenide channel waveguide," in Optics Express, vol. 23, no. 5, March 9, 2015.

[22] Pan Ma, Duk-Yong Choi, Yi Yu, Xin Gai, Zhiyong Yang, Sukanta Debbarma, Steve Madden and Barry Luther-Davies, "Low-loss chalcogenide waveguides for biosensing in the mid-infrared," 2014 IEEE Photonics Society Summer Topical Meeting Series, pp. 59-60, 2014.

[23] Pan Ma, Duk-Yong Choi, Yi Yu, Xin Gai, Zhiyong Yang, Sukanta Debbarma, Steve Madden and Barry Luther-Davies, "Low-loss chalcogenide waveguides for chemical sensing in the mid-infrared," in Optics Express, vol. 21, no. 24, December 2, 2013.

[24] R. Cherif, M. Zghal and L. Tartara, "Experimental and numerical analysis of white light generation in an air-silica photonic crystal fibre," 2009 3rd ICTON Mediterranean Winter Conference, pp. 1-6, 2009.

[25] Maxime Jacquet, Negative Frequency at the Horizon: Theoretical Study and Experimental Realisation of Analogue Gravity Physics in Dispersive Optical Media, Cham, Switzerland: Springer International Publishing, April 26, 2018.

[26] Ilaria Cristiani, Riccardo Tediosi, Luca Tartara and Vittorio Degiorgio, "Dispersive wave generation by solitons in microstructured optical fibers," in Optics Express, vol. 12, no. 1, January 12, 2004.

[27] William H. Press, Saul A. Teukolsky, William T. Vetterling and Brian P. Flannery, Numrical Recipes: The Art of Scientific Computiung, Third Edition, New York, USA: Cambridge University Press, September 10, 2007.

[28] Stephane Balac, Arnaud Fernandez, Fabrice Mahe, Florian Mehats, and Rozenn Texier-Picard, "The Interaction Picture Method for Solving the 
Generalized Nonlinear Schrödinger Equation in Optics," in ESAIM: Control, Optimisation and Calculus of Variations, vol. 50, no. 4, pp 945964, June 06, 2016.

[29] F. Matera, A. Mecozzi, M. Romagnoli, and M. Settembre, "Sideband instability induced by periodic power variation in long-distance fiber links," in Optics Letters, vol. 18, no. 18, September 15, 1993.

[30] B.M. Azizur Rahman and Arti Agrawal, Finite Element Modeling Methods for PHOTONICS, Norwood, Massachusetts, USA: Artech House, July 31, 2013.

[31] Mehrzad Tabatabaian, COMSOL for Engineers, Dulles, Virginia, UK: Mercury Learning and Information, March 27, 2014.

[32] Edmund J. F. Dickinson, Henrik Ekstrom and Ed Fontes, "Comsol Multiphysics: Finite element software for electrochemical analysis. A mini-review," in Electrochemistry Communications, vol. 40, pp. 71-74, March 2014.

[33] Michael Muller, Marco Kienel, Arno Klenke, Thomas Gottschall, Evgeny Shestaev, Marco Plotner, Jens Limpert and Andreas Tunnermann, "1 kW $1 \mathrm{~mJ}$ eight-channel ultrafast fiber laser," in Optics Letters, vol. 41, no. 15, August 1, 2016.

[34] Hudson D.D., Fuerbach A. and Jackson S.D., Progress in Mid-infrared Fiber Source Development, Singapore: Springer, 2018.

[35] Jie Ma, Zhipeng Qin, Guoqiang Xie, Liejia Qian and Dingyuan Tang, "Review of mid-infrared mode-locked laser sources in the $2.0 \mu \mathrm{m}-3.5 \mu \mathrm{m}$ spectral region," in Applied Physics Reviews, vol. 6, no. 2, June, 2019.

[36] V.Z. Kolev, M. W. Duering, B. Luther-Davies and A. V. Rode, "Compact high-power optical source for resonant infrared pulsed laser ablation and deposition of polymer materials," in Optics Express, vol. 14, no. 25, December 11, 2006.

[37] Y. Yu, X. Gai, C. Zhai, S. Qi, W. Guo, Z. Yang, R. Wang, D. Choi, S. Madden and B. Luther-Davies, "1.8-10 $\mu \mathrm{m}$ mid-infrared supercontinuum generation in a step-index chalcogenide fiber using low peak pump power," in Optical Letters, vol. 40, pp. 1081-1084, 2015.

[38] F. Silva, D.R. Austin, A. Thai, M. Baudisch, M. Hemmer, D. Faccio, A. Couairon and J. Biegert, "Multi-octave supercontinuum generation from mid-infrared filamentation in a bulk crystal," in Nature Communications, vol. 3, no. 807, 2012.

[39] K. A. Ingold, A. Marandi, M. J. F. Digonnet and R. L. Byer, "Sub-100fs fiber feedback synchronously pumped degenerate optical parametric oscillator," 2015 Conference on Lasers and Electro-Optics (CLEO), pp. 1-2, 2015.

[40] Yi Yu, Xin Gai, Ting Wang, Pan Ma, Rongping Wang, Zhiyong Yang, Duk-Yong Choi, Steve Madden and Barry Luther-Davies, "Mid-infrared supercontinuum generation in Chalcogenides," in Optical Materials Express, vol. 3, no. 8, August 1, 2013.

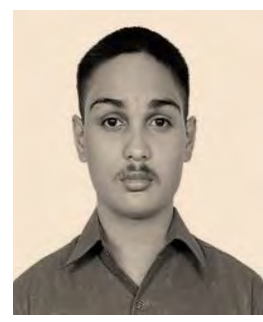

Mohammed Faisal was born in the port city, Chattogram of Bangladesh. He completed his A' Level and O' Level from Little Jewels School by the year 2016. He then completed his Bachelors of Science in Electrical and Electronics Engineering at Chittagong Independent University in the 2016-2021 session. He published a book named 'Control System Engineering Lab Manual: Simple and Concise' in 2019. He also presented his research work titled 'Study and Analysis of a Novel Hexagonal Core PCF for Broadband Supercontinuum Generation' in International Conference on Electronics, Communications and Information Technology 2021 (ICECIT 2021). He is an active researcher in the field of photonics.

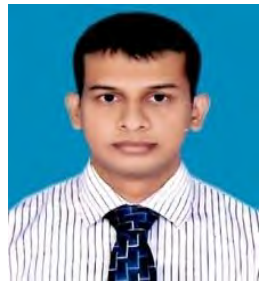

SK. M. Jahangir Kabir was born in 24 August,1993 in Chattogram, the port city of Bangladesh. He has completed his S.S.C from Chattogram Collegiate School in the year of 2008. Subsequently he has completed his H.S.C from Govt. Hazi Mohammaed Mohsin College, Chattogram in the year of 2010. He has completed his 2 years merchant naval training from Bangladesh Marine Academy in the year of 2013-2014 in Marine Engineering. Then he has completed his B.Sc. in Electrical and Electronics Engineering from Chittagong Independent University. His paper 'Study and Analysis of a Novel Hexagonal Core PCF for Broadband Supercontinuum Generation' has been presented on International Conference on Electronics, Communications and Information Technology 2021 (ICECIT 2021). He has a research interest is Photonics background.

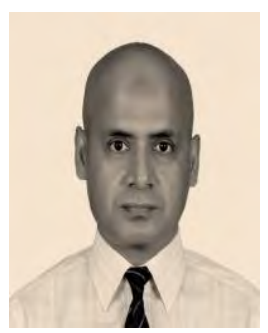

M. R. Karim completed his B.Sc in Electrical and Electronic Engineering from Khulna University of Engineering and Technology, Bangladesh in 1998 and then completed his M.Sc. in Electrical and Electronic Engineering from Bangladesh University of Engineering and Technology, Bangladesh in 2009. He accomplished his $\mathrm{PhD}$ from the University of London, UK and also attained a Post-Doctoral in Photonics from the University of Malaysia, Malaysia. Currently he is the Dean of the School of Science and Engineering at Chittagong Independent University. He has enormous amount of research papers and journals in his research background. He specializes in nonlinear optics and is an active researcher in the respective field. 